\title{
Tipifarnib and farnesyltransferase inhibitors in the treatment of inflammatory breast cancer: is the story over? A review
}

This article was published in the following Dove Press journal:

Orphan Drugs: Research and Reviews

10 May 2013

Number of times this article has been viewed

\author{
Irene Dehghan-Paz' \\ Dora Il'yasova ${ }^{2}$ \\ Kenneth L van Golen ${ }^{3}$ \\ Lydia Usha' \\ 'Division of Hematology and \\ Oncology, Rush University Medical \\ Center, Chicago, IL, USA; '2Duke \\ Cancer Institute, Duke University \\ Medical Center, Durham, NC, USA; \\ ${ }^{3}$ Department of Biological Sciences, \\ University of Delaware, Newark, DE, \\ USA
}

\begin{abstract}
Inflammatory breast cancer (IBC) is a rare but aggressive form of breast cancer with unique clinicopathological features and poor prognosis. Its epidemiology is distinct from noninflammatory locally advanced breast cancer (LABC), which points to different etiology. With the advent of multimodality treatment for LABC, the outcomes for women with IBC have improved, but remain significantly inferior to outcomes for noninflammatory LABC. This review focuses on new research in epidemiology and molecular pathways characteristic for IBC. One of the critical carcinogenic events apparently driving the development and progression of IBC is activation of the RhoC protein, which is a part of the Ras oncogene superfamily. These proteins, like other Ras proteins, require posttranslational prenylation for their activation and transfer to cell membranes. Farnesylation is a common type of prenlyation that can be blocked with a new class of drugs - farnesyltransferase inhibitors. The most advanced farnesyltransferase inhibitor in development, tipifarnib, has been evaluated in Phase II clinical trials as monotherapy and in combination with antihormonal agents and trastuzumab in metastatic and locally advanced breast cancer. A few tumor responses have been observed in these trials, but not enough to warrant a Phase III trial. Potential combinations of tipifarnib with other novel agents targeting enzymes downstream to RhoC are reviewed. Some of these drugs, such as imatinib and crizotinib, are already commercially available. Others like perifosine and anti-vascular endothelial growth factor 3 antibody are currently under development. Innovative trial designs to address this rare type of cancer are discussed.
\end{abstract}

Keywords: inflammatory breast cancer, farnesyltransferase inhibitors, tipifarnib, Rho proteins, clinical trials

\section{Introduction}

Inflammatory breast cancer (IBC) is a rare, aggressive, often fatal form of breast cancer (BC) that accounts for $1 \%-5 \%$ of newly diagnosed BCs in the US. ${ }^{1}$ IBC is not inflammatory, although the involved breast is erythematous and warm. Clinical features are due to numerous dermal tumor emboli in the papillary and reticular dermis of the skin overlying the breast. IBC is classified as a primary tumor, stage T4d. However, not all BCs with skin changes are IBCs.

\section{Etiology and epidemiology of inflammatory breast cancer}

The etiology of IBC is unknown. A role of genetic factors, including BRCAI and $B R C A 2$ mutations, has been suggested. ${ }^{2}$ Mathematical modeling of age-specific rates for IBC suggests different etiologies for IBC and for noninflammatory locally advanced 
breast cancer (LABC). They have different age-associated incidence patterns, and a different number of critical carcinogenic transition stages predicted by the model (6.3 for IBC, 8.5 for noninflammatory LABC). ${ }^{3}$ Data from Tunisia, Italy, and the US suggest a significant environmental/socioeconomic component in IBC etiology: more cases come from low socioeconomic status (SES) rural populations than from high-SES urban populations. In the US, African American women, a racial subgroup with lower SES, ${ }^{4}$ has around twofold higher rates of IBC compared to the racial subgroup with higher SES - Caucasian women. ${ }^{3,5,6}$ The diverging trends in the incidence of IBC and noninflammatory $\mathrm{BC}^{7}$ strongly suggest different etiologies. Moreover, sharp temporal changes in IBC occurrence (eg, in Tunisia, the proportion of BC classified as IBC decreased $\sim 9.5$-fold from $57 \%$ to $6 \%{ }^{7}$ ) point to environmental risk factors, including SES, because the genotype of the population cannot change that quickly. If these factors are identified, and if they are modifiable, the disease might be prevented.

In addition to temporal trends, analysis of Surveillance, Epidemiology and End Results (SEER) data in the US demonstrate an earlier age at diagnosis of IBC (peak $=51$ years) than of noninflammatory LABC (peak $=74$ years). This difference is also observed in other countries, and there are racial/ethnic disparities as well. ${ }^{5}$ However, these studies are ecological by study design, and therefore suffer from the insufficient individual-level characteristics required for the etiological analytical studies, such as cohort and case-control studies; nevertheless, they are important as hypothesis-generating studies. Analytical studies, particularly population-based case-control studies, are needed to establish etiological risk factors of IBC.

\section{Molecular characteristics of IBC}

Over the past decade, mounting evidence has indicated that IBC is not only clinically and histologically distinct from other forms of LABC but is also distinct at a molecular level. ${ }^{8,9}$ A number of signaling pathways have been identified that are unique to IBC. Many of these pathways were first delineated in the limited number of cell-culture and xenograft models that existed for IBC and then confirmed in patient samples. ${ }^{10}$ Ras homologous (Rho) C guanosine triphosphatase (GTPase) and lost in IBC (aka Wnt-inducible secreted protein 3) were the first proteins shown to be specifically altered in IBC versus non-IBC patient samples. ${ }^{11}$ Subsequently, several other molecular differences have been identified. For example, the platelet-derived growth-factor receptor alpha was found overexpressed in IBC patient samples compared with stage, grade, and cell type of originmatched non-IBC patient samples. ${ }^{8,10}$ Angiogenic and lymphangiogenic factors are upregulated in IBC as well. These factors support rapid growth of tumor cells under hypoxic conditions and also promote dissemination of tumor cells to distant sites. ${ }^{12-14}$ Amplification of anaplastic lymphoma kinase (ALK) is present in a majority of IBC patient tumors. ${ }^{15}$ ALK is a tyrosine kinase implicated in the pathogenesis of cancers such as neuroblastoma, non-small-cell lung carcinoma, and anaplastic large-cell lymphoma. ${ }^{16-18}$

\section{Clinical presentation and prognosis of IBC}

IBC has an unusual clinical presentation: (1) a more rapid onset (3-6 months), (2) breast redness, edema, excessive warmth, and pain (Figure 1), (3) more aggressive tumor characteristics than LABC (which usually manifests as a painless breast mass), (4) a peau d'orange (orange peel) appearance to the skin because of skin edema and exaggerated hair-follicle pits, ${ }^{19}$ (5) larger tumor sizes, (6) higher nuclear grades, and (7) more frequent involvement of

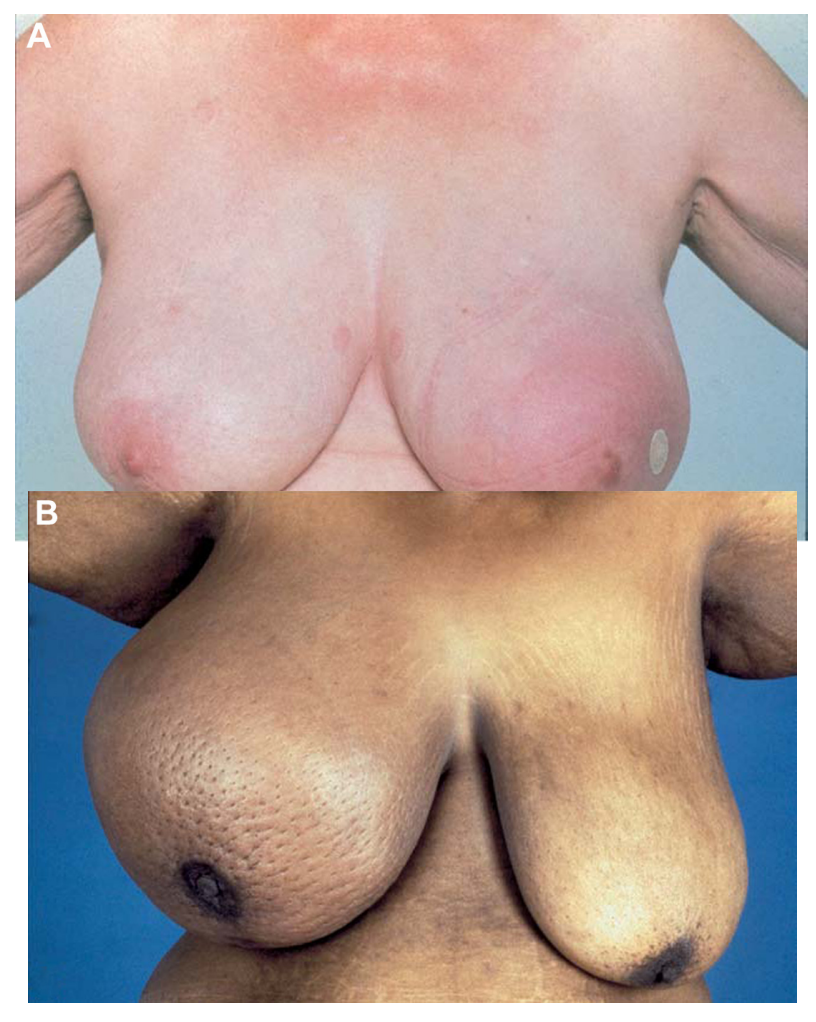

Figure I (A and B) Typical appearance of the breast in inflammatory breast cancer. ${ }^{21}$ (A) Breast erythema; (B) breast edema and enlargement with peau d'orange skin changes.

Reprinted from Seminars in Oncology, Volume 35, Singletary SE, Cristofanilli M, Defining the clinical diagnosis of inflammatory breast cancer, Pages 7-10, Copyright (C) 2008. ${ }^{21}$ 
lymph nodes. Approximately $30 \%$ of IBC patients have distant metastases at diagnosis. ${ }^{20}$

The presence of tumor emboli in the dermal lymphatic vessels is pathognomonic for $\mathrm{IBC}^{22}$ (Figure 2), which explains its unique clinical picture. It is observed on microscopic examination in $\sim 70 \%$ of cases,${ }^{23}$ and helps to make the diagnosis when present. However, IBC diagnosis is based on clinical findings, and the involvement of dermal lymphatics is not required. IBC is more likely to be hormone receptornegative and HER-2/neu tumor marker-positive than noninflammatory LABC. ${ }^{24-26}$

Despite improved outcomes due to multimodality therapy for BC in general, survival of women with IBC (5-year survival $41 \%$, median survival 2.9 years) is worse than for LABC (63\%, 6.4 years, respectively). ${ }^{5,24}$

\section{Standard multimodality therapy for IBC}

The standard-of-care treatment of IBC is neoadjuvant systemic therapy (NAST), followed by mastectomy and axillary lymph-node dissection, then post mastectomy radiation to the chest wall and adjuvant trastuzumab or hormone therapy if indicated. Data are limited regarding the optimal choice of chemotherapy, since IBC is a rare disease. ${ }^{19}$

Several chemotherapy regimens have been used in the neoadjuvant treatment of IBC, including a cyclophosphamide, methotrexate, and 5-fluorouracil regimen and a cyclophosphamide, doxorubicin, and 5-fluorouracil regimen, but it is unclear whether differences in these regimens affect overall

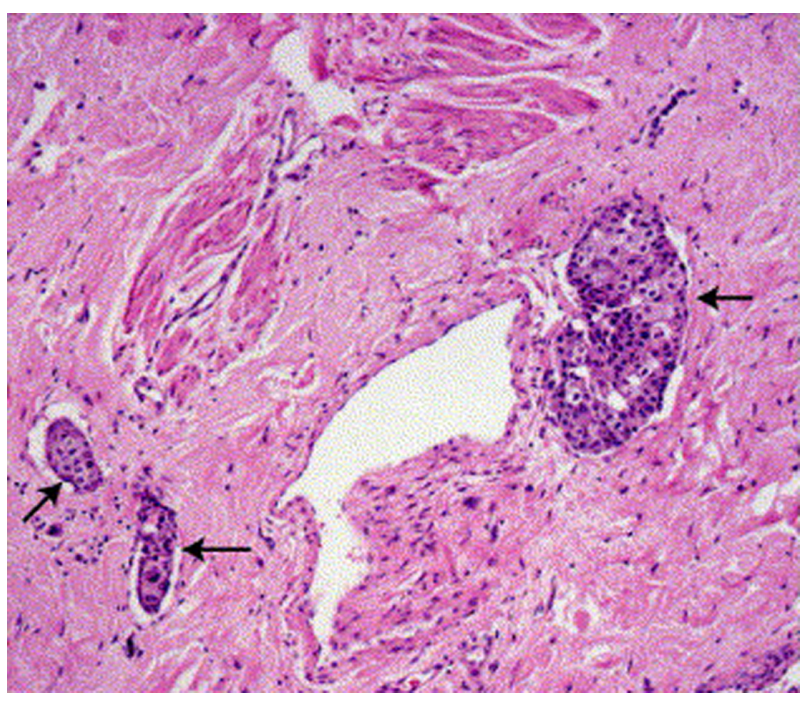

Figure 2 Histopathological evidence of subdermal lymphovascular invasion by cancer cells in inflammatory breast cancer. ${ }^{27}$

Note: Arrows indicate inflammatory breast cancer cells in dermal lymphatics. Reprinted from Surgical Oncology, Volume I4, Cariati M, Bennett-Britton TM, Pinder SE, Purushotham AD, “Inflammatory” breast cancer, Pages I33-143, Copyright (C) 2005. .7 $^{7}$ survival (OS). ${ }^{28}$ Adding paclitaxel to anthracycline-based therapy significantly improved patient outcomes for hormone receptor-negative IBC, with median OS and progression-free survival advantages of 22 and 9 months, respectively. ${ }^{29}$

In HER-2/neu-positive-IBC, a trastuzumab-based chemotherapy combination is used with most common regimens: (1) doxorubicin and cyclophosphamide followed by paclitaxel and trastuzumab; (2) docetaxel, carboplatin, and trastuzumab - trastuzumab is continued adjuvantly after mastectomy for 1 year. A subset analysis of IBC patients from a recent randomized Phase III study (the NOAH trial) found that adding trastuzumab to NAST improved the pathologic complete response (pCR) rate from $13 \%$ to $48 \% .^{30}$

Subsequent mastectomy after NAST followed by radiotherapy lowered the incidence of local recurrence, improved distant disease-free survival, and improved OS in patients who achieved a clinical complete or partial response to chemotherapy. ${ }^{31}$

Although multimodality therapy improved the prognosis of women with IBC, it remains inferior to prognosis in LABC. This has necessitated a search for novel agents to solve this important clinical problem. A promising new class of drugs that may help to address it is farnesyltransferase inhibitors (FTIs).

\section{Development of farnesyltransferase inhibitors against Ras-based tumors}

The Ras activated oncogene was the first human oncogene reported. The Ras protein belongs to a superfamily of about 100 proteins, including H-Ras, K-Ras, and N-Ras, which regulate cell proliferation. Of all cancers, $20 \%-25 \%$ harbor a mutation in the Ras GTPase oncogene, ${ }^{32}$ which leads to constitutively active expression of the Ras protein. Some cancers, like $\mathrm{BC}$, have a lower incidence of Ras mutations (5\%), while others such as pancreatic cancer have a much higher incidence of Ras mutations (90\%). ${ }^{33-35}$ Ras promotes growth-stimulatory signals via the mitogen-activated protein kinase pathway. ${ }^{36-49}$ Thus, actively targeting Ras proteins to prevent tumor growth has been a highly attractive approach. Ras, like other GTPases such as Rho proteins, requires posttranslational prenylation (a lipid-modification process with intermediates in the cholesterol biosynthesis pathway, such as the 15-carbon farnesyl and the 20-carbon geranylgeranyl groups) to be properly localized to the plasma membrane, become activated, and signal correctly. ${ }^{40-43}$ FTIs are the class of drugs rationally designed to inhibit farnesylation, and thus ras activation.

Ras is primarily farnesylated at the C-terminal end of the protein on a CAAX motif (cysteine, two aliphatic 
amino acids and any amino acid, Figure 3). ${ }^{41-43}$ The prenyl group is generated in the mevalonate pathway, the same pathway that produces cholesterol and dolichol. ${ }^{44}$ The farnesyl group is added to the cysteine of the CAAX motif via farnesyltransferase. ${ }^{45}$

A good deal of effort was put into understanding the mevalonate pathway and characterizing farnesyltransferase. ${ }^{45}$ The characterization of farnesyltransferase suggested its utility as a target. Thus, FTIs were originally developed to target tumors containing mutated Ras. ${ }^{46,47}$ Early FTIs blocked farnesylation of Ras in biochemical assays and in cells. ${ }^{46,48}$ Two classes of FTIs were developed: (1) direct inhibitors of farnesyltransferase and (2) farnesyltransferase substrates.

Preclinical experiments demonstrated that FTIs potently block Ras-mediated tumor cell growth. ${ }^{49-51}$ Initially, FTIs appeared to have great promise in Phase II clinical trials, demonstrating both potential efficacy and little toxicity. ${ }^{52-57}$ However, their performance in Phase III trials (Table 1) proved to be disappointing, ${ }^{56,58}$ which is not uncommon in oncologydrug development. The failure of FTIs against Ras-based tumors was suggested by a number of in vitro experiments as well. FTI resistance developed in some mutant K-Rascontaining cells. ${ }^{59,60}$ Other studies demonstrated that K- and N-Ras in FTI-treated cells can be alternately geranylgeranylated, with Ras activity remaining uninhibited. ${ }^{61-63}$

\section{Targeting Rho proteins with farnesyltransferase inhibitors}

Subsequently, attention turned to non-Ras targets, specifically the Rho GTPases, ${ }^{64,65}$ which comprise a subfamily of the Ras superfamily. The Rho proteins are 23 monomeric GTP-binding proteins that actively control all aspects of cytoskeletal reorganization. ${ }^{66,67}$ These GTP-binding proteins are inactive in their guanosine diphosphatase-bound form and active in their GTP-bound form. Early work showed a profound effect of FTIs on cytoskeletal morphology,

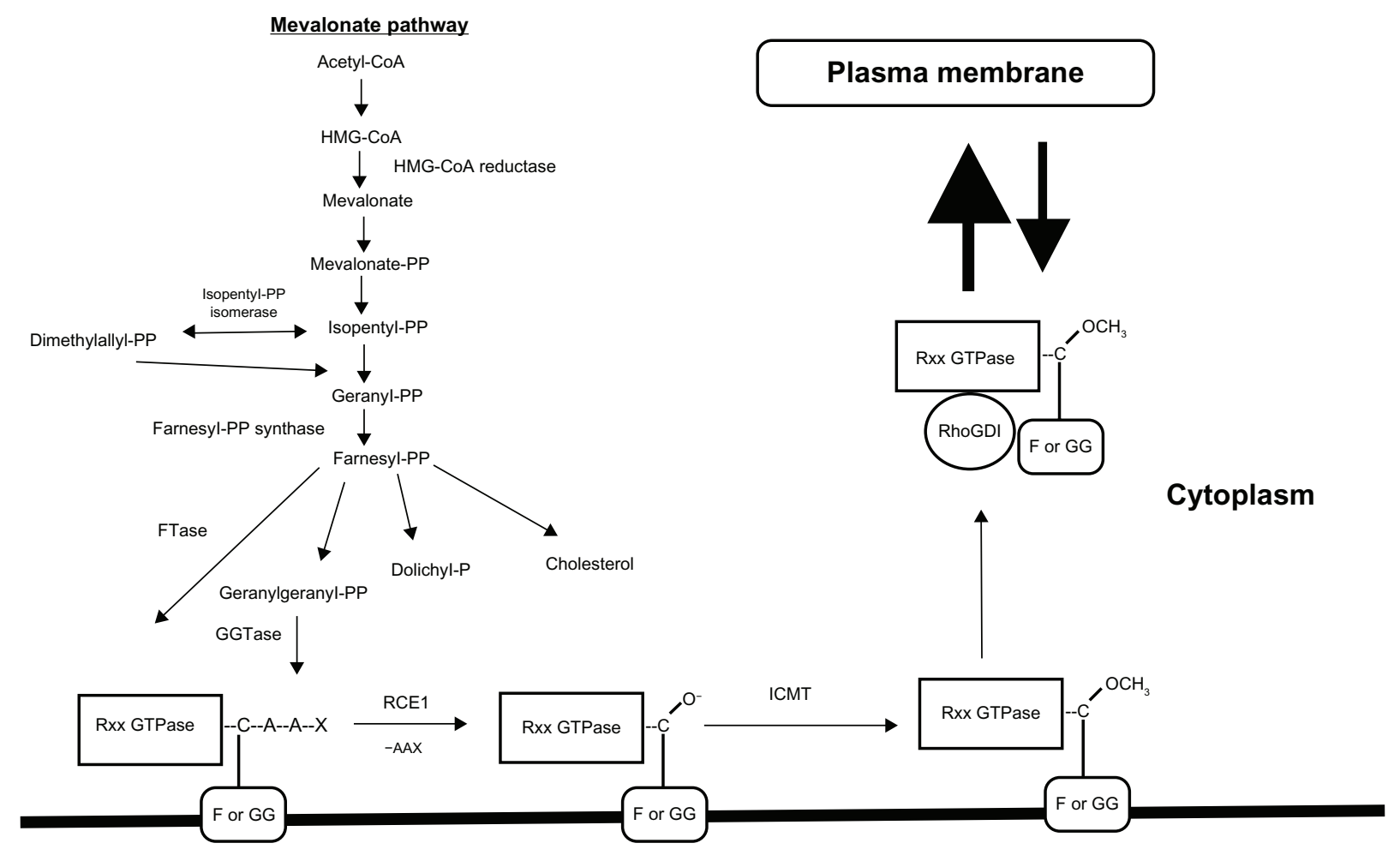

Endoplasmic reticulum

Figure 3 Prenylation of Ras and Rho guanosine triphosphatases (GTPases).

Notes: HMG-CoA is converted to mevalonate by HMG-CoA reductase, the rate-limiting step of the biosynthesis of isoprenoids. Mevalonate is converted to isopentyl-PP, which provides the basic five-carbon building block for the isoprenoids. Isopentyl-PP is converted to farnesyl-PP, a I5-carbon isoprenoid. Farneysl-PP can be converted to dolichyl-P, which is involved in $\mathrm{N}$-linked glycosylation, and in the metabolism of cholesterol or geranylgeranyl-PP, a 20-carbon isoprenoid. If the $\mathrm{X}$ of the -CAAX domain is a leucine or phenylalanine, then geranylgeranyltransferase I adds a geranylgeranyl-PP group to the GTPase. IF X is not a leucine or phenylalanine, then farnesyltransferase I adds a farnesyl-PP group to the protein. Ras-converting enzyme (RCEI) removes the-AAX group, and isoprenyl-cystiene-carboxymethyltransferase (ICMT) adds a carboxymethyl group. Prenylated Rho proteins are sequestered in the cytoplasm in their inactive GDP-bound form. Both prenylated Ras and Rho proteins are delivered to the plasma membrane, where the prenyl group is inserted into the lipid bilayer. Localization to the plasma membrane allows the GTPase to be activated.

Abbreviations: CoA, 3-hydroxy-3-methyl-glutaryl-CoA; F, farnesyl; GG, geranylgeranyl; GDP, guanosine diphosphate; GDI, guanosine diphosphate dissociation inhibitor; GGT, geranylgeranyl transferase; Rxx GTPase, Ras or Rho GTPase; PP, pyrophosphate. 
Table I Summary of Phase II/III trials with tipifarnib (excluding breast cancer trials) ${ }^{58,84-88}$

\begin{tabular}{|c|c|c|c|}
\hline Disease & Tipifarnib dose schedule & Main toxicities & Response/outcome \\
\hline \multicolumn{4}{|l|}{ Phase II } \\
\hline \multirow[t]{2}{*}{ Small-cell lung cancer (relapsed) ${ }^{84}$} & $400 \mathrm{mg}$ twice a day for days I-I4 every 3 weeks & Granulocytopenia & No significant activity \\
\hline & & Thrombocytopenia & \\
\hline Myeloproliferative disorders ${ }^{84}$ & $300 \mathrm{mg}$ twice a day for 21 days every 4 weeks & Thrombocytopenia & 2 complete responses \\
\hline \multirow[t]{3}{*}{ Pancreatic cancer ${ }^{84}$} & $300 \mathrm{mg}$ twice a day for 21 days every 4 weeks & Fatigue & No significant activity \\
\hline & & Malaise & \\
\hline & & Neutropenia & \\
\hline Glioma $^{84}$ & $300 \mathrm{mg}$ twice a day for 21 days every 4 weeks & Granulocytopenia & $3 / 33$ partial responses \\
\hline \multirow[t]{3}{*}{ Leukemias $^{84}$} & $600 \mathrm{mg}$ twice a day for 21 days every 4 weeks & Hypokalemia & $29 \%$ response rate \\
\hline & & Rash & \\
\hline & & Hyperbilirubinemia & \\
\hline \multirow[t]{2}{*}{ MDS, AML, CMML ${ }^{85}$} & $600 \mathrm{mg}$ twice a day for 21 days every $4-6$ weeks & Infection & I7\% complete responses \\
\hline & & Gastrointestinal & \\
\hline \multirow[t]{2}{*}{ Non-small-cell lung cancer ${ }^{84}$} & $300 \mathrm{mg}$ twice a day for 21 days every 4 weeks & Neutropenia & No partial or complete response \\
\hline & & Leukopenia & $14 \%$ stable disease \\
\hline \multirow[t]{4}{*}{ Advanced colorectal cancer ${ }^{86}$} & $300 \mathrm{mg}$ twice a day for 21 days every 4 weeks & Cytopenias & No significant activity \\
\hline & & Mood changes & \\
\hline & & Hyperbilirubinemia & \\
\hline & & Pneumonitis & \\
\hline \multirow[t]{3}{*}{ MDS $^{87}$} & $300 \mathrm{mg}$ twice a day for 21 days every 4 weeks & Anemia & $2 / 28$ complete responses \\
\hline & & Thrombocytopenia & I/28 partial response \\
\hline & & Neutropenia & \\
\hline \multicolumn{4}{|l|}{ Phase III } \\
\hline \multirow{4}{*}{$\begin{array}{l}\text { Pancreatic cancer } \\
\text { (gemcitabine }+ \text { [tipifarnib versus } \\
\text { placebo] })^{88}\end{array}$} & 200 mg twice a day continuously + gemcitabine & Neutropenia & No significant difference in \\
\hline & & Thrombocytopenia & overall survival \\
\hline & & Diarrhea & \\
\hline & & Hypokalemia & \\
\hline \multirow{3}{*}{ 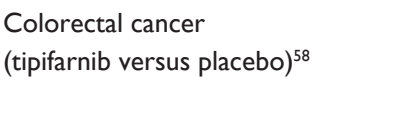 } & $300 \mathrm{mg}$ twice a day for 21 days every 4 weeks & Diarrhea & No significant difference in \\
\hline & & Rash & overall survival \\
\hline & & Myelosuppression & \\
\hline
\end{tabular}

Reproduced with permission of Kelland LR, Expert Opin Investig Drugs, 2003;12(3):4I3-421. Copyright @ 2003, Informa Healthcare. ${ }^{84}$

Abbreviations: MDS, myelodysplastic syndromes; AML, acute myelogenous leukemia; CMML, chronic myelomacrocytic leukemia.

thus suggesting an effect of this class of inhibitors on Rho function. ${ }^{49,59}$ Like Ras, Rho proteins are prenylated at their C-terminal end at the CAAX domain (Figure 3). Prenylation typically occurs as a farnesyl or geranylgeranyl group. ${ }^{42}$

\section{Preclinical studies of farnesyltransferase inhibitors in IBC}

Studies using the SUM149 IBC cell line were done to determine whether FTI treatment had an effect on the IBC cell line. SUM149 cells were developed from a primary triple-negative IBC and are of the basal B subtype ${ }^{68,69}$ The first direct comparison of IBC cells with non-IBC cells using a modified differential display and in situ hybridization identified RhoC GTPase as being overexpressed in $>90 \%$ of IBC cell lines and patient samples. ${ }^{11}$ RhoC GTPase was subsequently identified as a key facilitator of the metastatic IBC phenotype, primarily affecting cell motility and invasion (Figure 4). ${ }^{11,70,71}$ Furthermore, RhoC drives expression of proangiogenic factors, such as vascular endothelial growth factor. ${ }^{71}$ FTI treatment of IBC cells significantly reduced RhoC GTPaseinduced motility and invasion. Also, introduction of a mutant RhoB that can only be geranylgeranylated recapitulated FTI treatment. ${ }^{72}$

\section{Farnesyltransferase inhibitors induce dormancy in breast cancer cells}

The mechanisms of tumor cell dormancy are not well understood. Patients deemed "cured" of their disease can develop cancer recurrence years or decades later, with metastatic disease histologically identical to their original tumor. ${ }^{73}$ Treatment of IBC cells with FTI resulted in a phenotype similar to what is described for an in vitro model of dormancy. ${ }^{72} \mathrm{~A}$ recent study demonstrated that treatment of MCF-7 non-IBC cells with FTI L-422,831 also leads to a phenotype reminiscent of dormancy ${ }^{74,75}$ FTI treatment of the MCF-7 cell line leads to profound changes in Rho GTPase activation. ${ }^{74,75}$ Specifically, 
RhoA GTPase becomes hypoactivated, while RhoC GTPase becomes hyperactivated, producing radical changes in the cell cytoskeleton and morphology identical to that of FTI-treated SUM149 IBC cells. ${ }^{72}$

Similar to the in vitro model of dormancy, ${ }^{76,77}$ FTIinduced dormancy is reversible. ${ }^{72,75,78}$ Upon FTI withdrawal, cells grow normally after exiting from nearly 2 weeks of dormancy. ${ }^{75}$

\section{Implications for therapy}

Taken together, these studies suggest innovative approaches to the use of FTIs in IBC. Potentially, FTI-induced dormancy could be exploited to make IBC cells more susceptible to conventional chemotherapies by synchronizing the cell cycle. Upon FTI withdrawal, dividing cells could be targeted with chemotherapy. The combination of statins with FTIs could decrease de novo synthesis of prenyl groups and prevent farnesylation of proteins by exogenous isoprenes. A molecular signature of RhoC expression and activation along with active Akt1 in the tumor would be predictors of patients that would benefit from FTI clinical trials. Current evidence suggests that FTIs could be paired with other known compounds that target pathways important in the IBC phenotype. Examples of such compounds include phosphatidylinositide kinase 3/Akt inhibitors such as perifosine, platelet-derived growth-factor receptor inhibitors such as imatinib, and ALK inhibitors.

\section{Clinical experience with farnesyltransferase inhibitors in malignancy}

A number of FTIs have been evaluated in Phase I, Phase II, and even Phase III clinical trials, including tipifarnib, lonafarnib, salirasib, L778123, and BMS-214662. ${ }^{79}$ Although some of them are still under investigation, none has been approved by the Food and Drug Administration (FDA) for clinical use.

\section{Tipifarnib as an antineoplastic agent}

Tipifarnib (Zarnestra, R115777, Johnson and Johnson) (Figure 5) is the best-studied FTI. It has been evaluated against both hematologic malignancies and solid tumors (Table 1).

In Phase I trials against hematologic malignancies, the tipifarnib doses tested ranged from 100 to $1200 \mathrm{mg}$ twice a day (BID). In a Phase I trial of poor-risk leukemia, the dose-limiting toxicity was neurotoxicity, which occurred at $1200 \mathrm{mg}$ BID. ${ }^{80}$ No dose-response relationships were

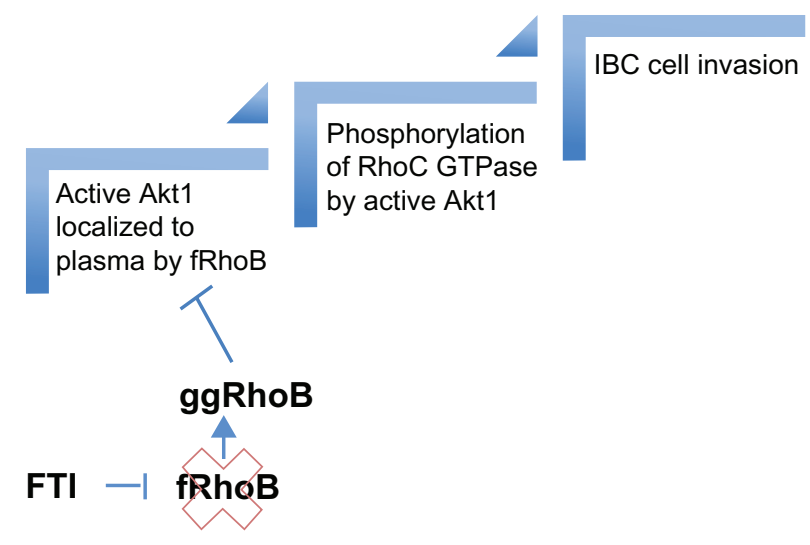

Figure 4 Proposed schema for farnesyltransferase inhibitor (FTI) action in inflammatory breast cancer (IBC) cells.

Notes: Aktl is localized to the plasma membrane via farnesylated RhoB guanosine triphosphatase (GTPase) (fRhoB). Aktl phosphorylates RhoC GTPase, promoting IBC cell invasion. FTI inhibits farnesylation of RhoB, shifting the population towards geranylgeranylated RhoB (ggRhoB), and relocalizes Aktl, preventing RhoC phosphorylation. RhoC can become active, but does not drive invasion. Abbreviations: $\mathrm{fRhoB}$, farnesylated RhoB; ggRoB, geranylgeranylated RhoB.

seen, and CRs were seen at the lowest dose level of $100 \mathrm{mg}$ BID. ${ }^{80}$ In a Phase I trial of tipifarnib in myelodysplastic syndromes, more than $75 \%$ inhibition of farnesyltransferase was observed in peripheral mononuclear cells at all doses. ${ }^{81}$

In Phase I studies in solid tumors, the maximum tolerated dose ranged from 300 to $500 \mathrm{mg}$ BID. ${ }^{79}$ Similar to the hematologic malignancies, the frequent dose-limiting toxicity of tipifarnib was neurotoxicity as well as myelosuppression. ${ }^{82,83}$

Based on the suboptimal outcomes of Phase III trials (Table 1), tipifarnib has not been approved by the FDA for any clinical indication, although it continues to be investigated in acute myelogenous leukemia.

\section{Tipifarnib in breast cancer}

Of the various Phase II trials that have evaluated tipifarnib against solid tumors, BC studies predominate. Tipifarnib has been evaluated in Phase II studies as monotherapy, in combination with chemotherapy, in combination with hormonal

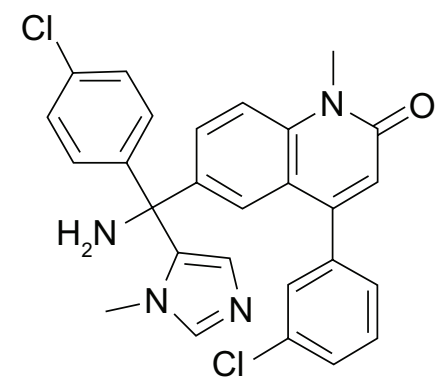

Figure $\mathbf{5}$ Skeletal structure of tipifarnib. 
therapy, and in a Phase I study in combination with an antiHER-2/neu-targeted agent trastuzumab. ${ }^{89}$

In 2003, Johnston et al published the results of a single-agent tipifarnib study that compared two cohorts of advanced $\mathrm{BC}$ patients treated with either continuous dosing $(C D, n=41)$ of tipifarnib 400 or $300 \mathrm{mg}$ BID or intermittent dosing (ID, $\mathrm{n}=35$ ) of $300 \mathrm{mg}$ BID for 21 days every 4 weeks. ${ }^{57}$ In the CD cohort, $10 \%$ had a partial response and $15 \%$ had stable disease at or beyond 24 weeks, whereas in the ID cohort, 14\% had a partial response and $9 \%$ had stable disease. Due to grade $3 / 4$ neutropenia seen initially at the $400 \mathrm{mg}$ BID dose in the CD cohort, most patients were treated with tipifarnib $300 \mathrm{mg}$ BID. ${ }^{57}$ Toxicities - mainly neutropenia, thrombocytopenia, and neurotoxicity - were less frequent in the ID cohort. Tipifarnib showed clinical activity as a single agent for treatment of metastatic BC, and ID was superior CD.

In 2009, Sparano et al evaluated the effect of tipifarnib on the pCR rate. Tipifarnib was added to a neoadjuvant doxorubicin-cyclophosphamide regimen being given to patients with clinical stage IIB-IIIC breast cancer. ${ }^{90}$ Of the total 44 patients, eleven $(25 \%)$ had a breast $\mathrm{pCR}$. This was greater than the $10 \%-15 \%$ pCR rate in historical controls treated with doxorubicin-cyclophosphamide chemotherapy alone..$^{90}$ In the course of the treatment, farnesyltransferase enzyme activity in the tumor was decreased in most patients. Twelve patients with IBC were included in this study; among them, two (17\%) achieved breast $\mathrm{pCR} .{ }^{90}$ The authors concluded that tipifarnib inhibits farnesyltransferase activity in vivo and enhances the breast pCR rate, which warrants further evaluation. Among biomarkers studied in this trial was expression of RhoA, -B, and $-\mathrm{C}$ GTPase proteins in the tumors. The investigators concluded that there was no association between Rho protein expression and tumor response. However, as discussed earlier, it is the Rho activity, not expression, that drives the tumor phenotype. This is particularly true for RhoC GTPase, where high expression levels are toxic to the cell. Also, the prenylation state of RhoB GTPase, ie, a shift in farnesylated to geranylgeranylated RhoB upon FTI treatment, was not assessed in the study. The prenylation state is likely to be the key factor determining tumor responses to FTIs and it is independent of protein expression levels.

More recently, in 2012, a Phase II trial looked at the addition of capecitabine to tipifarnib in 63 anthracyclinetreated and taxane-resistant metastatic BC patients. The objective response rate was $9.5 \%$, compared to a historical response rate of up to $25 \%$ for capecitabine alone at a higher dose in this patient population. ${ }^{91}$ The conclusion was that the tipifarnib-capecitabine combination was not more effective than capecitabine alone in metastatic BC. ${ }^{91}$

In addition to tipifarnib-chemotherapy combinations, tipifarnib was evaluated in combinations with hormonal agents such as letrozole, fulvestrant, and tamoxifen. There were no significant differences in response rate, response duration, time to disease progression, or survival in these studies. $^{92-94}$

Thus, no Phase II trials with tipifarnib alone or in combination with other drugs showed enough efficacy to proceed with a Phase III BC trial. However, some activity of tipifarnib as a single agent was demonstrated in metastatic $\mathrm{BC}$ and in combination with standard neoadjuvant chemotherapy in LABC. ${ }^{57,90}$ As discussed earlier, few IBC patients were included in the latter trial, and their outcomes were analyzed as a separate subset without significant difference between IBC and noninflammatory BC.$^{90}$ Unfortunately, the sample was too small to draw any meaningful conclusions. No studies specifically addressed IBC. Based on the unique clinical and molecular characteristics of IBC, we propose that tipifarnib still holds promise in IBC if combined with novel targeted agents, and/or if an improved trial design is implemented.

\section{A clinical trial design to study the effect of tipifarnib or another FTI in IBC}

Trials specifically addressing IBC are rarely conducted. Most relevant clinical trials are for $\mathrm{LABC}$ : women with IBC may be allowed to participate, or quite often they are excluded because of IBC's unique biology and poor prognosis. ${ }^{19}$ Nevertheless, since NAST is the standard of care for IBC, there is an opportunity to see a potential pathologic response quite soon after initiation of treatment, when the patient is going for a planned mastectomy. Until recently, a pCR after neoadjuvant chemotherapy could not be the basis for accelerated drug approval. However, in May 2012, the FDA issued new guidelines to allow using pCR in NAST of high-risk early stage $\mathrm{BC}$ as an end point to support accelerated approval. ${ }^{95}$ Since IBC progresses rapidly, it is potentially dangerous to expose women with IBC to an experimental agent that may be ineffective while withholding established therapy. Therefore, an FTI must be combined with a standard anthracycline- or paclitaxel-based NAST to increase its efficacy, as measured by the $\mathrm{pCR}$ rate. This design was utilized in the already-discussed Phase II LABC trial, ${ }^{90}$ which allowed enrollment of IBC patients. A difficulty with this approach is the absence of a control arm. Thus, historical controls are used, and any effect must be quite robust to further pursue drug development. 
Neoadjuvant trials combining standard chemotherapy, such as paclitaxel with novel treatments in $\mathrm{LABC}$, are still a good venue for studying novel drugs in IBC, provided IBC patients are enrolled and stratified to differentiate their outcomes from the outcomes for non-IBC LABC patients.

As mentioned earlier, FTI withdrawal may increase cancer sensitivity to chemotherapy. This suggests that a short course of pretreatment with an FTI may potentiate the effect of NAST with doxorubicin and cyclophosphamide followed by paclitaxel.

With the advent of characterizing disease based on molecular subtypes, increasingly clinical trials use an adaptive design, which is more flexible and allows one to change therapy to another drug quickly. ${ }^{96}$ However, a paucity of IBC patients is still a barrier. A solution may be a national or international clinical trial, which has been a recent trend in oncology. The disadvantage of international trials is the use of different clinical standards across the globe, especially in staging.

Aside from the neoadjuvant setting, novel drugs can be studied in the adjuvant setting when residual IBC is present after standard neoadjuvant treatment. Because of the poor prognosis of women who have not achieved a complete response, this approach is appealing since it would fill an unmet need. On the other hand, this design requires a control arm and a longer period of observation.

Finally, an FTI can be studied in metastatic IBC. The study design should incorporate a tissue reassessment after drug exposure. A recently opened Eastern Cooperative Oncology Group trial-E2108: A randomized Phase III trial of the value of early local therapy for the intact primary tumor in patients with metastatic breast cancer ${ }^{97}$ - has an attractive design in IBC. Patients with IBC are eligible for this study.

In view of limitations inherent to conducting clinical trials in this rare disease, it is hard to overestimate the importance of preclinical research with accurate target identification and development of IBC animal models. An improved understanding of "molecular drivers" in IBC would certainly help in optimizing the clinical trial design and in decreasing the required patient sample size in this rare cancer. ${ }^{98,99}$ It is critical to identify a molecular signature allowing for IBC patient selection for FTI treatment. Such a signature, the RASGRP1:APTX geneexpression ratio, was suggested for acute myeloid leukemia where it was associated with a high likelihood of response to FTIs. $^{79}$

\section{Summary}

The class of FTIs was rationally developed with highthroughput screening to inhibit the expression of the Ras oncogene, one of the most commonly activated oncogenes in human cancers. Since the Ras oncogene requires posttranslational prenylation for its activation, blocking this process was a rational goal in the attempt to suppress cancer cell proliferation. Although preclinical studies with FTIs were encouraging, clinical trials were disappointing. Tipifarnib is the most advanced FTI. Nonetheless, it is not FDA-approved for clinical use.

Initially, BC was an unlikely malignancy to benefit from tipifarnib, because Ras mutations are uncommon in $\mathrm{BC}$. Surprisingly, however, Phase II trials ${ }^{90-93}$ with tipifarnib in $\mathrm{BC}$ were promising. This may be the result of inhibiting farnesylation of other enzymes, not necessarily of Ras. Over 300 proteins in human cells require farnesylation for their activation. ${ }^{40}$ Unfortunately, the anticancer activity of tipifarnib was deemed insufficient to pursue a Phase III trial in BC. No trials have studied the potential role of tipifarnib specifically in IBC.

Recent research has identified new molecular targets in IBC, including RhoC in the Rho subfamily of Ras proteins, which also requires prenylation (typically, farnesylation) for its activation. ${ }^{8}$ Activation of RhoC may be a key step in IBC carcinogenesis. Thus, revisiting the potential of tipifarnib in IBC is warranted.

The importance of prenylation in activation of key molecules in IBC was underscored by the recent retrospective study showing prolongation of disease-free survival in women with IBC who have taken hydrophilic statins at the time of diagnosis. ${ }^{100}$

To be able to better identify a potential benefit for patients with IBC, we propose use of improved clinical trial designs for (1) specifically addressing this rare disease, (2) studying the activity of RhoC before and after treatment with tipifarnib, (3) analyzing the activity of other farnesylated proteins in search of other possible targets, and (4) combining tipifarnib with agents inhibiting molecules downstream to Ras and RhoC.

Some of the candidate drugs to combine with tipifarnib or another FTI for the treatment of IBC are commercially available, while others are investigational. Among commercially available agents is imatinib, one of the first targeted agents, which is FDA-approved for the treatment of chronic myelogenous leukemia and gastrointestinal stromal tumors. Also, an ALK inhibitor, crizotinib, was recently approved for treating ALK-positive non-small-cell lung cancer. Investigational agents include an Akt inhibitor, perifosine, which was studied in Phase II trials against advanced renal cell carcinoma and the anti-vascular endothelial growth factor 3 monoclonal antibody IMC-3C5, which specifically inhibits lymphangiogenesis, ${ }^{101,102}$ a very important target in IBC. 
Obviously, these novel combinations need to be evaluated first in preclinical models with IBC cell lines such as SUM149 and with a xenograft model. If these studies confirm the synergism between two agents, a Phase I/II trial would be warranted. Given the rarity of IBC, it may not be feasible to conduct a trial just for IBC patients. However, allowing them to participate in neoadjuvant, metastatic, and adjuvant (when residual disease is present) trials along with separate analysis of their outcomes will facilitate the development of new drugs for IBC.

It is not uncommon in medicine that medications originally designed to treat one disease or a group of diseases find application to a different group of diseases. Unexpectedly, FTIs were found to hold promise for patients with another rare disease - Hutchinson-Gilford progeria syndrome (HGPS) - a genetic condition causing affected children to age and die prematurely. This disease is characterized by production of a permanently farnesylated mutant lamin A protein termed progerin. ${ }^{103}$ FTI-227 reverses cellular defects in Hutchinson-Gilford progeria syndrome cells in vitro. ${ }^{104,105}$ FTIs are currently in clinical trials for HGPS.

\section{Acknowledgments}

The authors are very grateful to Jeremy Fields, $\mathrm{PhD}$, for thoughtful review of the manuscript, to Ms Jonna Peterson, MLIS, for comprehensive literature search, and to Ms Jill McHugh for the valuable assistance in manuscript preparation.

\section{Disclosure}

The authors report no conflicts of interest in this work.

\section{References}

1. Levine PH, Steinhorn SC, Ries LG, Aron JL. Inflammatory breast cancer: the experience of the Surveillance, Epidemiology, and End Results (SEER) program. J Natl Cancer Inst. 1985;74(2):291-297.

2. Jimenez AM, Growney A, Behrens G, Corbridge C, Chapman DD, Usha L. Hereditary inflammatory breast cancer associated with BRCA2 mutation: a rare disease presentation in mother and daughter. Clin Adv Hematol Oncol. 2012;10(6):402-404.

3. Il'yasova D, Siamakpour-Reihani S, Akushevich I, Akushevich L, Spector N, Schildkraut J. What can we learn from the age- and race/ ethnicity-specific rates of inflammatory breast carcinoma? Breast Cancer Res Treat. 2011;130(2):691-697.

4. Ward E, Jemal A, Cokkinides V, et al. Cancer disparities by race/ ethnicity and socioeconomic status. CA Cancer J Clin. 2004;54(2): 78-93.

5. Hance KW, Anderson WF, Devesa SS, Young HA, Levine PH. Trends in inflammatory breast carcinoma incidence and survival: the Surveillance, Epidemiology, and End Results program at the National Cancer Institute. J Natl Cancer Inst. 2005;97(13):966-975.

6. Chang S, Parker SL, Pham T, Buzdar AU, Hursting SD. Inflammatory breast carcinoma incidence and survival: the Surveillance, Epidemiology, and End Results program of the National Cancer Institute, 1975-1992. Cancer. 1998;82(12):2366-2372.
7. Boussen H, Bouzaiene H, Ben Hassouna J, et al. Inflammatory breast cancer in Tunisia: epidemiological and clinical trends. Cancer. 2010;116(Suppl 11):2730-2735.

8. Joglekar M, Golen KL. Molecules that drive the invasion and metastasis of inflammatory breast cancer. In: Ueno NT, Fink T, editors. Inflammatory Breast Cancer: An Update. New York: Springer; 2012:161-184.

9. Radunsky GS, van Golen KL. The current understanding of the molecular determinants of inflammatory breast cancer metastasis. Clin Exp Metastasis. 2005;22(8):615-620.

10. Lacerda L, Woodward WA. Models of inflammatory breast cancer. In: Ueno NT, Fink T, editors. Inflammatory Breast Cancer: An Update. New York: Springer; 2012:139-150.

11. van Golen KL, Davies S, Wu ZF, et al. A novel putative low-affinity insulin-like growth factor-binding protein, LIBC (lost in inflammatory breast cancer), and RhoC GTPase correlate with the inflammatory breast cancer phenotype. Clin Cancer Res. 1999;5(9):2511-2519.

12. Van der Auwera I, Van den Eynden GG, Colpaert CG, et al. Tumor lymphangiogenesis in inflammatory breast carcinoma: a histomorphometric study. Clin Cancer Res. 2005;11(21):7637-7642.

13. Van der Auwera I, Van Laere SJ, Van den Eynden GG, et al. Increased angiogenesis and lymphangiogenesis in inflammatory versus noninflammatory breast cancer by real-time reverse transcriptase-PCR gene expression quantification. Clin Cancer Res. 2004;10(23):7965-7971.

14. Vermeulen PB, van Golen KL, Dirix LY. Angiogenesis, lymphangiogenesis, growth pattern, and tumor emboli in inflammatory breast cancer: a review of the current knowledge. Cancer. 2010;116(Suppl 11):2748-2754.

15. Tuma RS. ALK gene amplified in most inflammatory breast cancers. J Natl Cancer Inst. 2012;104(2):87-88.

16. Kwak EL, Bang YJ, Camidge DR, et al. Anaplastic lymphoma kinase inhibition in non-small-cell lung cancer. $N$ Engl J Med. 2010;363(18):1693-1703.

17. Chiarle R, Voena C, Ambrogio C, Piva R, Inghirami G. The anaplastic lymphoma kinase in the pathogenesis of cancer. Nat Rev Cancer. 2008;8(1):11-23.

18. Decock A, Ongenaert M, Vandesompele J, Speleman F. Neuroblastoma epigenetics: from candidate gene approaches to genome-wide screenings. Epigenetics. 2011;6(8):962-970.

19. Robertson FM, Bondy M, Yang W, et al. Inflammatory breast cancer: the disease, the biology, the treatment. CA Cancer J Clin. 2010;60(6):351-375.

20. Anderson WF, Schairer C, Chen BE, Hance KW, Levine PH. Epidemiology of inflammatory breast cancer (IBC). Breast Dis. 2005;22:9-23.

21. Singletary SE, Cristofanilli M. Defining the clinical diagnosis of inflammatory breast cancer. Semin Oncol. 2008;35(1):7-10.

22. Bonnier $P$, Charpin $C$, Lejeune $C$, et al. Inflammatory carcinomas of the breast: a clinical, pathological, or a clinical and pathological definition? Int J Cancer. 1995;62(4):382-385.

23. Bertucci F, Finetti P, Rougemont J, et al. Gene expression profiling for molecular characterization of inflammatory breast cancer and prediction of response to chemotherapy. Cancer Res. 2004;64(23):8558-8565.

24. Cristofanilli M, Valero V, Buzdar AU, et al. Inflammatory breast cancer (IBC) and patterns of recurrence: understanding the biology of a unique disease. Cancer. 2007;110(7):1436-1444.

25. Paradiso A, Tommasi S, Brandi M, et al. Cell kinetics and hormonal receptor status in inflammatory breast carcinoma: comparison with locally advanced disease. Cancer. 1989;64(9):1922-1927.

26. Turpin E, Bieche I, Bertheau P, et al. Increased incidence of ERBB2 overexpression and TP53 mutation in inflammatory breast cancer. Oncogene. 2002;21(49):7593-7597.

27. Cariati M, Bennett-Britton TM, Pinder SE, Purushotham AD. "Inflammatory" breast cancer. Surg Oncol. 2005;14(3):133-143.

28. Harris EE, Schultz D, Bertsch H, Fox K, Glick J, Solin LJ. Ten-year outcome after combined modality therapy for inflammatory breast cancer. Int J Radiat Oncol Biol Phys. 2003;55(5):1200-1208.

29. Cristofanilli M, Gonzalez-Angulo AM, Buzdar AU, Kau SW, Frye DK, Hortobagyi GN. Paclitaxel improves the prognosis in estrogen receptor negative inflammatory breast cancer: the M D Anderson Cancer Center experience. Clin Breast Cancer. 2004;4(6):415-419. 
30. Baselga J, Semiglazov V, Manikhas GM, et al. Efficacy of neoadjuvant trastuzumab in patients with inflammatory breast cancer: data from the NOAH (neoadjuvant herceptin) phase III trial [abstract 2030]. Eur $J$ Cancer. 2007;5:193.

31. Fleming RY, Asmar L, Buzdar AU, et al. Effectiveness of mastectomy by response to induction chemotherapy for control in inflammatory breast carcinoma. Ann Surg Oncol. 1997;4(6):452-461.

32. Downward J. Targeting RAS signalling pathways in cancer therapy. Nat Rev Cancer. 2003;3(1):11-22.

33. Almoguera C, Shibata D, Forrester K, Martin J, Arnheim N, Perucho M. Most human carcinomas of the exocrine pancreas contain mutant c-Kras genes. Cell. 1988;53(4):549-554.

34. Smit VT, Boot AJ, Smits AM, Fleuren GJ, Cornelisse CJ, Bos JL. KRAS codon 12 mutations occur very frequently in pancreatic adenocarcinomas. Nucleic Acids Res. 1988;16(16):7773-7782.

35. Rochlitz CF, Scott GK, Dodson JM, et al. Incidence of activating ras oncogene mutations associated with primary and metastatic human breast cancer. Cancer Res. 1989;49(2):357-360.

36. Gospodarowicz D, Brown KD, Birdwell CR, Zetter BR. Control of proliferation of human vascular endothelial cells: characterization of the response of human umbilical vein endothelial cells to fibroblast growth factor, epidermal growth factor, and thrombin. $J$ Cell Biol. 1978;77(3):774-788.

37. Hall A, Marshall CJ, Spurr NK, Weiss RA. Identification of transforming gene in two human sarcoma cell lines as a new member of the ras gene family located on chromosome 1. Nature. 1983;303(5916): 396-400.

38. Brown R, Marshall CJ, Pennie SG, Hall A. Mechanism of activation of an N-ras gene in the human fibrosarcoma cell line HT1080. EMBO J. 1984;3(6):1321-1326.

39. Feramisco JR, Gross M, Kamata T, Rosenberg M, Sweet RW. Microinjection of the oncogene form of the human H-ras (T-24) protein results in rapid proliferation of quiescent cells. Cell. 1984;38(1):109-117.

40. Rowinsky EK. Lately, it occurs to me what a long, strange trip it's been for the farnesyltransferase inhibitors. J Clin Oncol. 2006;24(19):2981-2984.

41. Kim R, Rine J, Kim SH. Prenylation of mammalian ras protein in xenopus oocytes. Mol Cell Biol. 1990;10(11):5945-5949.

42. Adamson P, Marshall CJ, Hall A, Tilbrook PA. Post-translational modifications of p21rho proteins. J Biol Chem. 1992;267(28):20033-20038.

43. Cox AD, Der CJ. Protein prenylation: more than just glue? Curr Opin Cell Biol. 1992;4(6):1008-1016.

44. Buhaescu I, Izzedine H. Mevalonate pathway: a review of clinical and therapeutical implications. Clin Biochem. 2007;40(9-10):575-584.

45. Manne V, Roberts D, Tobin A, et al. Identification and preliminary characterization of protein-cysteine farnesyltransferase. Proc Natl Acad Sci U S A. 1990;87(19):7541-7545.

46. Gibbs JB, Oliff A, Kohl NE. Farnesyltransferase inhibitors: Ras research yields a potential cancer therapeutic. Cell. 1994;77(2):175-178.

47. Reiss Y, Goldstein JL, Seabra MC, Casey PJ, Brown MS. Inhibition of purified p21ras farnesyl:protein transferase by cys-AAX tetrapeptides. Cell. 1990;62(1):81-88.

48. Das NP, Allen CM. Inhibition of farnesyl transferases from malignant and non-malignant cultured human lymphocytes by prenyl substrate analogues. Biochem Biophys Res Commun. 1991;181(2):729-735.

49. Prendergast GC, Davide JP, deSolms SJ, et al. Farnesyltransferase inhibition causes morphological reversion of ras-transformed cells by a complex mechanism that involves regulation of the actin cytoskeleton. Mol Cell Biol. 1994;14(6):4193-4202.

50. Cox AD, Garcia AM, Westwick JK, et al. The CAAX peptidomimetic compound B581 specifically blocks farnesylated, but not geranylgeranylated or myristylated, oncogenic ras signaling and transformation. J Biol Chem. 1994;269(30):19203-19206.

51. Kohl NE, Wilson FR, Mosser SD, et al. Protein farnesyltransferase inhibitors block the growth of ras-dependent tumors in nude mice. Proc Natl Acad Sci U S A. 1994;91(19):9141-9145.
52. Adjei AA, Erlichman C, Davis JN, et al. A phase I trial of the farnesyl transferase inhibitor SCH66336: evidence for biological and clinical activity. Cancer Res. 2000;60(7):1871-1877.

53. Eskens FA, Awada A, Cutler DL, et al. Phase I and pharmacokinetic study of the oral farnesyl transferase inhibitor SCH 66336 given twice daily to patients with advanced solid tumors. J Clin Oncol. 2001;19(4):1167-1175.

54. Punt CJ, van Maanen L, Bol CJ, Seifert WF, Wagener DJ. Phase I and pharmacokinetic study of the orally administered farnesyl transferase inhibitor R115777 in patients with advanced solid tumors. Anticancer Drugs. 2001;12(3):193-197.

55. Sharma S, Kemeny N, Kelsen DP, et al. A phase II trial of farnesyl protein transferase inhibitor SCH 66336, given by twice-daily oral administration, in patients with metastatic colorectal cancer refractory to 5-fluorouracil and irinotecan. Ann Oncol. 2002;13(7):1067-1071.

56. Adjei AA, Mauer A, Bruzek L, et al. Phase II study of the farnesyl transferase inhibitor R115777 in patients with advanced non-small-cell lung cancer. J Clin Oncol. 2003;21(9):1760-1766.

57. Johnston SR, Hickish T, Ellis P, et al. Phase II study of the efficacy and tolerability of two dosing regimens of the farnesyl transferase inhibitor, R115777, in advanced breast cancer. J Clin Oncol. 2003; 21(13):2492-2499.

58. Rao S, Cunningham D, de Gramont A, et al. Phase III double-blind placebo-controlled study of farnesyl transferase inhibitor R115777 in patients with refractory advanced colorectal cancer. J Clin Oncol. 2004;22(19):3950-3957.

59. Prendergast GC, Davide JP, Lebowitz PF, Wechsler-Reya R, Kohl NE. Resistance of a variant ras-transformed cell line to phenotypic reversion by farnesyl transferase inhibitors. Cancer Res. 1996;56(11):2626-2632.

60. James G, Goldstein JL, Brown MS. Resistance of K-RasBV12 proteins to farnesyltransferase inhibitors in Rat1 cells. Proc Natl Acad Sci USA. 1996;93(9):4454-4458.

61. Whyte DB, Kirschmeier P, Hockenberry TN, et al. K- and N-ras are geranylgeranylated in cells treated with farnesyl protein transferase inhibitors. J Biol Chem. 1997;272(22):14459-14464.

62. Zhang FL, Kirschmeier P, Carr D, et al. Characterization of ha-ras, $\mathrm{N}$-ras, ki-Ras4 A, and ki-Ras4B as in vitro substrates for farnesyl protein transferase and geranylgeranyl protein transferase type I. J Biol Chem. 1997;272(15):10232-10239.

63. Rowell CA, Kowalczyk JJ, Lewis MD, Garcia AM. Direct demonstration of geranylgeranylation and farnesylation of ki-ras in vivo. $J$ Biol Chem. 1997;272(22):14093-14097.

64. Lebowitz PF, Prendergast GC. Non-ras targets of farnesyltransferase inhibitors: focus on rho. Oncogene. 1998;17(11 Reviews): 1439-1445.

65. Lebowitz PF, Davide JP, Prendergast GC. Evidence that farnesyltransferase inhibitors suppress ras transformation by interfering with rho activity. Mol Cell Biol. 1995;15(12):6613-6622.

66. Wennerberg K, Der CJ. Rho-family GTPases: it's not only rac and rho (and I like it). J Cell Sci. 2004;117(Pt 8):1301-1312.

67. Wheeler AP, Ridley AJ. Why three rho proteins? RhoA, RhoB, RhoC, and cell motility. Exp Cell Res. 2004;301(1):43-49.

68. Du W, Lebowitz PF, Prendergast GC. Cell growth inhibition by farnesyltransferase inhibitors is mediated by gain of geranylgeranylated RhoB. Mol Cell Biol. 1999;19(3):1831-1840.

69. Liu A, Du W, Liu JP, Jessell TM, Prendergast GC. RhoB alteration is necessary for apoptotic and antineoplastic responses to farnesyltransferase inhibitors. Mol Cell Biol. 2000;20(16):6105-6113.

70. van Golen KL, Wu ZF, Qiao XT, Bao LW, Merajver SD. RhoC GTPase, a novel transforming oncogene for human mammary epithelial cells that partially recapitulates the inflammatory breast cancer phenotype. Cancer Res. 2000;60(20):5832-5838.

71. van Golen KL, Wu ZF, Qiao XT, Bao L, Merajver SD. RhoC GTPase overexpression modulates induction of angiogenic factors in breast cells. Neoplasia. 2000;2(5):418-425. 
72. van Golen KL, Bao L, DiVito MM, Wu Z, Prendergast GC, Merajver SD. Reversion of RhoC GTPase-induced inflammatory breast cancer phenotype by treatment with a farnesyl transferase inhibitor. Mol Cancer Ther. 2002;1(8):575-583.

73. Sosa MS, Bragado P, Debnath J, Aguirre-Ghiso JA. Regulation of tumor cell dormancy by tissue microenvironments and autophagy. $A d v$ Exp Med Biol. 2013;734:73-89.

74. Chaterjee M, van Golen KL. Breast cancer stem cells survive periods of farnesyl-transferase inhibitor-induced dormancy by undergoing autophagy. Bone Marrow Res. 2011;2011:362938.

75. Chatterjee M, van Golen KL. Farnesyl transferase inhibitor treatment of breast cancer cells leads to altered RhoA and RhoC GTPase activity and induces a dormant phenotype. Int J Cancer. 2011;129(1):61-69.

76. Barrios J, Wieder R. Dual FGF-2 and intergrin alpha5beta1 signaling mediate GRAF-induced RhoA inactivation in a model of breast cancer dormancy. Cancer Microenviron. 2009;2(1):33-47.

77. Barrios J, Wieder R. FGF-2-induced breast cancer dormancy in an in vitro model is maintained through integrin alpha5beta1 signaling. $\mathrm{Am}$ Assoc Cancer Res. 2007:100.

78. Chatterjee M, van Golen KL. RhoA and RhoC GTPases in dormancy of breast cancer. Proceedings of the 100th Annual Meeting of the American Association of Cancer Research; 2009 Apr 18-22; Denver, CO, USA.

79. Tsimberidou AM, Chandhasin C, Kurzrock R. Farnesyltransferase inhibitors: where are we now? Expert Opin Investig Drugs. 2010;19(12):1569-1580.

80. Karp JE, Lancet JE, Kaufmann SH, et al. Clinical and biologic activity of the farnesyltransferase inhibitor R115777 in adults with refractory and relapsed acute leukemias: a phase 1 clinical-laboratory correlative trial. Blood. 2001;97(11):3361-3369.

81. Kurzrock R, Kantarjian HM, Blascovich MA, et al. Phase I study of alternate-week administration of tipifarnib in patients with myelodysplastic syndrome. Clin Cancer Res. 2008;14(2):509-514.

82. Crul M, de Klerk GJ, Swart M, et al. Phase I clinical and pharmacologic study of chronic oral administration of the farnesyl protein transferase inhibitor R115777 in advanced cancer. J Clin Oncol. 2002;20(11):2726-2735

83. Zujewski J, Horak ID, Bol CJ, et al. Phase I and pharmacokinetic study of farnesyl protein transferase inhibitor R115777 in advanced cancer. J Clin Oncol. 2000;18(4):927-941.

84. Kelland LR. Farnesyl transferase inhibitors in the treatment of breast cancer. Expert Opin Investig Drugs. 2003;12(3):413-421.

85. Lancet J, Gotlib J, Gojo I, et al. Tipifarnib (Zarnestra) in previously untreated poor-risk AML of the elderly: updated results of a multicenter phase 2 trial. Blood. 2004;104:249a.

86. Whitehead RP, McCoy S, Macdonald JS, et al. Phase II trial of R115777 (NSC \#70818) in patients with advanced colorectal cancer: a Southwest Oncology Group study. Invest New Drugs. 2006;24(4):335-341.

87. Kurzrock R, Albitar M, Cortes JE, et al. Phase II study of R115777, a farnesyl transferase inhibitor, in myelodysplastic syndrome. $J$ Clin Oncol. 2004;22(7):1287-1292.

88. Van Cutsem E, van de Velde H, Karasek P, et al. Phase III trial of gemcitabine plus tipifarnib compared with gemcitabine plus placebo in advanced pancreatic cancer. J Clin Oncol. 2004;22(8):1430-1438.

89. Schwartz G, Rowinsky E, Rha S, et al. A phase I, pharmacokinetic and biologic correlative study of R115777 and trastuzumab (herceptin) in patients with advanced cancer. Proc Am Soc Clin Oncol. 2001;20:322

Orphan Drugs: Research and Reviews

\section{Publish your work in this journal}

Orphan Drugs: Research and Reviews is an international, peer-reviewed open access journal publishing original research, reports, reviews and commentaries on all areas of the design and development of orphan drugs for the treatment of rare diseases through to clinical applications. Clinical outcomes, patient safety, and programs for the development and
90. Sparano JA, Moulder S, Kazi A, et al. Phase II trial of tipifarnib plus neoadjuvant doxorubicin-cyclophosphamide in patients with clinical stage IIB-IIIC breast cancer. Clin Cancer Res. 2009; 15(8):2942-2948.

91. Li T, Guo M, Gradishar WJ, et al. A phase II trial of capecitabine in combination with the farnesyltransferase inhibitor tipifarnib in patients with anthracycline-treated and taxane-resistant metastatic breast cancer: an Eastern Cooperative Oncology Group study (E1103). Breast Cancer Res Treat. 2012;134(1):345-352.

92. Johnston SR, Semiglazov VF, Manikhas GM, et al. A phase II, randomized, blinded study of the farnesyltransferase inhibitor tipifarnib combined with letrozole in the treatment of advanced breast cancer after antiestrogen therapy. Breast Cancer Res Treat. 2008;110(2):327-335.

93. Li T, Christos PJ, Sparano JA, et al. Phase II trial of the farnesyltransferase inhibitor tipifarnib plus fulvestrant in hormone receptor-positive metastatic breast cancer: New York Cancer Consortium trial P6205. Ann Oncol. 2009;20(4):642-647.

94. Dalenc F, Doisneau-Sixou SF, Allal BC, et al. Tipifarnib plus tamoxifen in tamoxifen-resistant metastatic breast cancer: a negative phase II and screening of potential therapeutic markers by proteomic analysis. Clin Cancer Res. 2010;16(4):1264-1271.

95. Prowell TM, Pazdur R. Pathological complete response and accelerated drug approval in early breast cancer. $N$ Engl J Med. 2012; 366(26):2438-2441.

96. Gupta S, Faughnan ME, Tomlinson GA, Bayoumi AM. A framework for applying unfamiliar trial designs in studies of rare diseases. J Clin Epidemiol. 2011;64(10):1085-1094.

97. Eastern Cooperative Oncology Group. E2108 - A randomized phase III trial of the value of early local therapy for the intact primary tumor in patients with metastatic breast cancer. 2012. Available from: http://ecog. dfci.harvard.edu/general/E2108info.html. Accessed February 9, 2013.

98. Atkinson JM, Gilbertson RJ. Developing treatment strategies for rare cancers. Oncotarget. 2011;2(9):657.

99. Casali PG. Do rare cancers deserve specific strategies for cancer research? Lancet Oncol. 2010;11(6):506-507.

100. Brewer T, Masuda H, Iwamoto T, et al. Statin use and improved survival outcome in primary inflammatory breast cancer: retrospective cohort study. Paper presented at the San Antonio Breast Cancer Symposium; December 4-8, 2012; San Antonio, TX, USA.

101. Kurenova EV, Hunt DL, He D, et al. Vascular endothelial growth factor receptor-3 promotes breast cancer cell proliferation, motility and survival in vitro and tumor formation in vivo. Cell Cycle. 2009;8(14):2266-2280.

102. Martell R, Chiorean E, Youssoufian H, et al. Phase I study of the antiVEGFR-3 monoclonal antibody IMC-3C5 in subjects with advanced solid tumors refractory to standard therapy or for which no standard therapy is available. J Clin Oncol. 2011;29 Suppl:TPS150.

103. Reddy S, Comai L. Lamin A, farnesylation and aging. Exp Cell Res. 2012;318(1):1-7.

104. Mehta IS, Bridger JM, Kill IR. Progeria, the nucleolus and farnesyltransferase inhibitors. Biochem Soc Trans. 2010;38(Pt 1):287-291.

105. Capell BC, Erdos MR, Madigan JP, et al. Inhibiting farnesylation of progerin prevents the characteristic nuclear blebbing of Hutchinson-Gilford Progeria syndrome. Proc Natl Acad Sci U S A. 2005;102(36):12879-12884. 